\title{
Symmetry-breaking in the independent particle model: Nature of the singular behavior of Hartree-Fock potentials
}

\author{
Josef Paldus · Tokuei Sako · Xiangzhu Li · Geerd H. F. Diercksen
}

\author{
J. Paldus $(\otimes)$ \\ Department of Applied Mathematics, University of Waterloo, Waterloo, Ontario, Canada N2L 3G1; \\ also at Department of Chemistry and Guelph-Waterloo Center for Graduate Work in Chemistry \\ $(\mathrm{GWC})^{2}$ - Waterloo Campus, University of Waterloo, Waterloo, Ontario, Canada N2L 3G1 \\ e-mail: paldus@uwaterloo.ca \\ T. Sako \\ Laboratory of Physics, College of Science and Technology, Nihon University, 7-24-1 Narashinodai, \\ Funabashi, 274-8501 Chiba, Japan \\ e-mail: sako@phys.ge.cst.nihon-u.ac.jp \\ web-page: http://www.phys.ge.cst.nihon-u.ac.jp/sako/ \\ X. Li \\ Department of Applied Mathematics, University of Waterloo, Waterloo, Ontario, Canada N2L 3G1 \\ e-mail: xli@scienide2.uwaterloo.ca \\ G. H. F. Diercksen \\ Max-Planck-Institut für Astrophysik, Karl-Schwarzschild-Strasse 1, D-85741 Garching, Germany \\ e-mail: ghd@mpa-garching.mpg.de \\ web-page: http://www.mpa-garching.mpg.de/mol_physics/index.shtml
}




\begin{abstract}
The nature of the singular behavior of Hartree-Fock (HF) potential energy surfaces (PESs) that arises in the presence of a spin-preserving instability of the relevant restricted HF solutions is illustrated by a simple $\pi$-electron model of the allyl radical as described by the Pariser-Parr-Pople (PPP) semi-empirical Hamiltonian. The simplicity of this three-electron model system stems from a low dimension of the appropriate variational space which enables an independent direct analytical approach illustrating the appropriateness of doublet stability conditions for restricted openshell HF (ROHF) solutions. At the same time it permits the derivation of explicit expressions for the energy providing a complete description of swallowtail or Whitneyfold catastrophe singularities on the corresponding PES that arise with the onset of a doublet instability. In particular, this simple model enables the computation of the part of the PES that is associated with unstable ROHF solutions and which would be difficult if not impossible to generate in full generality via standard self-consistent field $(\mathrm{SCF})$ iterative procedures in more complex situations.
\end{abstract}

Keywords Restricted open-shell Hartree-Fock (ROHF) solutions · Doublet instability · Pariser-Parr-Pople Hamiltonian · Allyl radical $\pi$-electron model · Symmetry breaking · Potential energy surfaces · Swallowtail or Whitney-fold singularity 


\section{Introduction}

The Hartree-Fock (HF) approximation is undoubtedly the most often exploited version of the independent particle model (IPM) and represents nowadays a standard tool in investigations of the atomic and molecular electronic structure. Although such a description is often lacking in accuracy due to an average account of the interelectronic Coulomb interactions, it nonetheless provides a useful qualitative - and even semi-quantitative - description of various molecular properties and serves as a point of departure for most post-HF correlated methods.

The relevant wave function $|\Phi\rangle$ has the form of a single anti-symmetrized product of molecular orbitals (MOs) or spin-orbitals (MSOs) $\left|A_{i}\right\rangle$,

$$
|\Phi\rangle=\mathfrak{A}\left|A_{1} A_{2} \cdots A_{N}\right\rangle,
$$

where $\mathfrak{A}$ designates an appropriate anti-symmetrizer and distinguishes itself by yielding the "best" IPM energy $E[\Phi]$,

$$
E[\Phi]=\langle\Phi|H| \Phi\rangle, \quad\langle\Phi \mid \Phi\rangle=1 .
$$

The HF equations are then obtained by requiring the first variation of the energy mean value functional $E[\Phi]$, Eq. (2), to vanish, i.e.,

$$
\left.\delta^{(1)} E[\Phi]\right|_{\Phi=\Phi_{0}}=0, \quad\left\langle\Phi_{0} \mid \Phi_{0}\right\rangle=1,
$$

where $\left|\Phi_{0}\right\rangle$ designates the desired HF solution.

It is often assumed that this stationary point on the mean energy hyper-surface $E[\Phi]$, Eqs. (2) and (3), represents the global minimum. This is indeed the case in many instances, at least when investigating standard molecular systems in the vicinity of their equilibrium geometry. However, when considering entire potential energy surfaces (PESs) or curves (PECs), even for atoms and diatomics, this is no longer the case (see, e.g., [1-17]; for reviews see [18-21]). Yet, the fact that $E\left[\Phi_{0}\right]$ represents a stationary point on $E[\Phi]$ is no guarantee that it represents the absolute minimum or, in fact, even a local minimum. In order to find out at least the local character of such a stationary point we have to examine the second variation, $\delta^{(2)} E\left[\Phi_{0}\right]$, leading to Thouless' stability conditions [22]. The positive definiteness of $\delta^{(2)} E\left[\Phi_{0}\right]$ then provides a sufficient condition for a $\mathrm{HF}$ solution to represent a local minimum on $E[\Phi]$. Such solutions are referred to as stable ones in contrast to unstable solutions in which case $\delta^{(2)} E\left[\Phi_{0}\right]<0$ (the case when $\delta^{(2)} E\left[\Phi_{0}\right]=0$ is more complex; see, e.g., [15] and references therein). Such unstable HF solutions are thus characterized by stationary points representing a maximum or a saddle point on $E[\Phi]$. Consequently, in the presence of an instability another HF solution having a lower energy than the unstable one must exist and no potential barrier on $E[\Phi]$ will separate it from the unstable solution. This is clearly warranted by the fact that the energy functional $E[\Phi]$ decreases in some direction in the variational space from the stationary point characterizing an unstable solution and that any HF solution represents an upper bound to the exact energy.

Within the standard MSO-based, finite-dimensional, quantum-chemical models relying on the linear combination of atomic orbitals (LCAO) approximation the Thouless' stability conditions take the form of a corresponding Hessian. In such a case it is 
very important to precisely specify the variational manifold involved. Specifically, for Hamiltonians that are invariant with respect to various symmetry group operations (spatial, spin, alternancy, particle number, etc.) we can formulate corresponding symmetry restricted $\mathrm{HF}$ solutions [e.g., restricted $\mathrm{HF}$ (RHF) solutions with doubly occupied MOs or unrestricted HF (UHF) solutions with different spin-up and spindown MOs for closed-shell systems or the so-called restricted open-shell HF (ROHF) solutions for simple open-shells, etc.] and corresponding stability conditions. In the case of an overall spin symmetry this leads to the so-called singlet and non-singlet or triplet stability conditions [18,19,23-26] for closed-shells, to doublet stability conditions for simple open-shells [27,28] or, generally, for high-spin HF solutions [14]. These conditions and the issuing broken-symmetry (BS) solutions were explored for a large number of systems, both at the semi-empirical $[12,13,23,25,26,29,30]$ and ab initio $[1-4,6-11,14,15,17]$ levels. The other types of symmetry restricted stability conditions were explored by Fukutome [18,31-35], providing a general classification for closed-shell type systems later extended to open-shells [21]. In particular, we mention several extensive reviews on this topic [18-21].

At this point it is important to emphasize that both stable, symmetry-adapted as well as BS HF solutions - the latter arising in the presence of an instability of the symmetric solution - employ the same symmetry-adapted Hamiltonian. Consequently, these BS solutions are invariably degenerate (in case of binary symmetry operations doubly degenerate) and we shall refer to them as BS solutions of the first kind. This fact is also closely related with the so-called Löwdin symmetry dilemma [36], even though in this case the symmetric and BS solutions may both be stable in principle and separated by a potential barrier. The implications and usefulness of BS solutions will be discussed in greater detail below. Nonetheless, the presence of an instability implies the existence of a BS HF solution with lower energy and, ultimately, of a $B S$ solution of the second kind that breaks the symmetry of the Hamiltonian (i.e., of the nuclear framework) with yet lower energy.

Let us now point out that the symmetry breaking at the IPM level (e.g., for HF or Brueckner-type solutions [37]) does not necessarily reflect the reality of the relevant molecular structures implied by BS solutions of the second kind since it may only indicate the inadequacy of the IPM arising due to the lack of correlation effects. In such cases the instability and the corresponding BS solutions may disappear when the correlation effects are properly accounted for and a complete basis set limit is approached (cf., e.g., [38]). Even when the symmetry breaking persists at the correlated level, this effect may be extremely small to be verified experimentally (cf., e.g., the results for the $\mathrm{BNB}$ and $\mathrm{N}_{3}$ species $[9,11]$ ). Nonetheless, even though the symmetry breaking may not appear at the equilibrium geometry, it will manifest itself sooner or later away from the equilibrium. This is the case, for example, that arises even for homonuclear diatomics $[14,15]$ in which case the symmetry breaking may lead to asymmetric dissociation products. Yet, in many cases the implied symmetry breaking is real, leading to the so-called Wigner molecules (Wigner dimerization, Wigner lattice, crystals, islands, bubbles, stripes or even Wigner necklase). This is particularly the case for extended systems leading, for example, to bond-length alternation in polyenic chains (or their cyclic polyene models, see, e.g., [5, 18, 23, 24, 
39-43]). A nice model example which is also clearly understood on physical grounds is the $\mathrm{H}_{6}$ model system [4].

It is the purpose of this paper to present a simple example involving an openshell system which clearly and explicitly displays the nature of a singular behavior of the relevant PES or PEC that arises in the presence of doublet instability. For this purpose we employ the Pariser-Parr-Pople (PPP) model of the allyl radical [30]. The stability of its ROHF solutions was also explored at the ab initio level $[1,2]$. This example will also enable us to compare general doublet stability conditions [24] with a directly generated analytical result by relying on a simple, one-parametric form of the energy functional for this model.

\section{Broken-symmetry solutions vs. broken-symmetry structures}

At this stage it is important to make a clear distinction between two kinds of $\mathrm{HF}$ solutions that are often referred to as BS solutions, namely those obtained with a fully symmetry-adapted Hamiltonian and those that employ a BS Hamiltonian based on an appropriately distorted molecular structure, the latter implying a BS equilibrium geometry or simply a distortion along some vibrational coordinate. We shall refer to them as BS solutions of the first and second kind, respectively. In the first instance the BS RHF or ROHF solution arises due to the presence of a spinpreserving instability (singlet instability in a closed-shell case or doublet instability in a simple open-shell case). We shall focus here on solutions preserving not only the total spin component $S_{z}$ but also the total spin $S^{2}$, i.e., on RHF or ROHF solutions. The total-spin-violating solutions that arise in the presence of a triplet or a nonsinglet instability in closed-shell systems lead to UHF solutions that break the $S^{2}$ invariance and often also the space symmetry (as is, e.g., the case for quantum dots; see reviews $[44,45])$. Clearly, open-shell systems are always unstable to total-spin breaking in view of spin-polarization due to a different number of spin-up and spindown electrons. It is worth noting that the doublet stability for simple open-shells (or, generally, total-spin preserving high-spin ROHF solutions for general open-shell systems) are absent in Fukutome's classification [31-35] that is restricted to closedshell systems. A more general classification, including ROHF solutions, was given in [21].

In view of the nonlinearity of HF equations we can often find several BS solutions of the first kind (for a general search for multiple HF solutions, see [46]) that arise even in the case of spin-preserving symmetry breaking (i.e., those arising as a consequence of singlet or doublet instabilities in the presence of multiple negative roots in the relevant stability problem; cf. [3]). Such BS solutions are not necessarily stable and thus lead to new BS solutions. For example, in the case of cyclic polyenes $\mathrm{C}_{N} \mathrm{H}_{N}, N=2 n=4 \nu+2$, $\nu=1,2, \cdots$ as described by the semi-empirical PPP Hamiltonian such pure singlet BS RHF solutions may display two types of charge density waves (CDW) which have been referred to as the diagonal and off-diagonal CDWs [23-26] (see also [28,30]). In the former case these solutions display alternating atomic charges but possess identical bond-orders while the latter case is characterized by alternating bond-orders and standard uniform unit atomic charges. The BS solutions with diagonal CDWs are again singlet unstable and lead to stable bond-order alternating solutions with 
off-diagonal CDWs. Similarly, in the atomic case of a doubly negative oxygen $\mathrm{O}^{2-}$ we can generate two closed-shell BS solutions having the symmetry of an oblate and a prolate symmetric top, the former one being again singlet unstable [6] (note that these solutions arise because of the "confinement" due to the use of finite basis sets; more information in this regard may be found in $[6,10])$. In general, multiple BS solutions are found whenever the HF stability problem yields several negative $\operatorname{roots} \lambda_{i}$, the corresponding eigenvectors pointing to various stationary points or HF solutions on the mean energy hyper-surface $E[\Phi]$ (see, e.g., $[14,15]$ for more detail). The stable BS solution usually corresponds to the lowest eigenvalue $\lambda_{i}$.

Now, when a BS solution of the first kind breaks the spatial symmetry of the symmetry-adapted Hamiltonian by displaying a symmetry-breaking (e.g., off-diagonal) CDW, we can actually arrive to BS solutions of the second kind by correspondingly distorting the molecular geometry to an appropriate subgroup of the original fullysymmetric point group. For example, in the case of cyclic polyenes, the distorted geometry will be characterized by the $D_{n h}$ point group in lieu of the $D_{N h}$ group. Clearly, such BS solutions of the first kind are degenerate being related via the symmetry group operation that is missing in the subgroup (in the $\mathrm{C}_{N} \mathrm{H}_{N}$ case this will be the $C_{N}$ rotation so that the BS solution displays the bond-order alternation along the chain).

Thus, when we consider an electronic Hamiltonian that is associated with a distorted geometry of the nuclear framework (alternating bond-lengths in the $\mathrm{C}_{N} \mathrm{H}_{N}$ case) the BS solutions of the second kind will arise splitting the degeneracy of the BS solutions of the first kind. Generally, the in-phase deformation (shorter bond-length for larger bond-orders and vice versa) will lower the energy while the out-of-phase deformation will increase it (see, e.g., [5]). It can be shown that the potential energy curves as a function of the distortion parameter (e.g., the difference or the ratio of longer and shorter bond lengths in $\mathrm{C}_{N} \mathrm{H}_{N}$ ) will cross at a finite angle precisely at the energy of the degenerate BS solutions associated with the undistorted geometry (cf. $[4,5,19,20,39])$. Yet, the potential associated with the out-of-phase deformation will generally exist in only a limited range of small deformations.

An unstable, symmetry-adapted solution may also be, in principle, extended to broken-symmetry geometries. Yet, the actual computation of such solutions via careful "analytic continuation" can only be achieved in a very small interval of distorted geometries even though such a solution must exist within the above mentioned range given by the out-of-phase type potentials with higher energy than the BS solution of the first kind. This is of course associated with the fact that these unstable solutions correspond to a maximum or a saddle point on the mean energy hyper-surface $E[\Phi]$ in which case the iterative self-consistent field (SCF) procedure will invariably collapse to a stable, lower-lying solution.

The general nature of PESs or PECs of the kind arising in the presence of a spinpreserving symmetry breaking has been briefly pointed out earlier $[4,5,9,17]$ but, due to the above explained difficulties, these potentials have never been explicitly generated in full detail. In the closed-shell case the general form of this type of a singular behavior was described by Mestechkin (see Fig. 1 of [20]) using perturbation theory with distortions along the symmetric and asymmetric vibrational coordinates 
employing a rather formidable formalism. In either case it can be shown that the resulting PES or PEC displays what is known in the catastrophe theory as a swallowtail or Whitney-fold singularity. Yet, as already pointed out it is very difficult to generate such a PES computationally. We thus present a simple model system in which case the nature and the origin of such a singular behavior can be easily generated and displayed.

\section{Stability conditions}

\subsection{General Thouless stability conditions}

The Thouless stability conditions [22] in a spin-orbital form may be easily derived by considering the second variation of the mean energy functional $E[\Phi]$ at the HF solution $|\Phi\rangle=\left|\Phi_{0}\right\rangle$ which can be expressed as a Hermitian quadratic form (see, e.g., $[19,21,23])$

$$
\delta^{(2)} E=\frac{1}{2}\left[\begin{array}{l}
\mathbf{C} \\
\overline{\mathbf{C}}
\end{array}\right]^{\dagger}\left[\begin{array}{ll}
\mathbf{A} & \mathbf{B} \\
\overline{\mathbf{B}} & \overline{\mathbf{A}}
\end{array}\right]\left[\begin{array}{l}
\mathbf{C} \\
\overline{\mathbf{C}}
\end{array}\right],
$$

with $\mathbf{A}^{\dagger}=\mathbf{A}$ and $\mathbf{B}^{\dagger}=\overline{\mathbf{B}}$. Here $\mathbf{C}$ is a column matrix of coefficients $c_{A_{i} \rightarrow A^{i}}$ that are associated with mono-excitations $A_{i} \rightarrow A^{i}$. The subscripts and superscripts indicate MSOs that are, respectively, occupied and unoccupied in the HF wave function $\left|\Phi_{0}\right\rangle=$ $\mathfrak{A}\left|A_{1}, A_{2}, \cdots, A_{N}\right\rangle$. A dagger indicates the Hermitian conjugate quantity and a bar the complex conjugation. Further, A represents the CI submatrix within the manifold of monoexcitations and $\mathbf{B}$ the block between the ground and biexcited states, i.e.,

$$
\begin{aligned}
A_{A_{i} \rightarrow A^{i}, A_{j} \rightarrow A^{j}} & =f_{A^{i}, A^{j}} \delta_{A_{j}, A_{i}} f_{A_{j}, A_{i}} \delta_{A^{i}, A^{j}}+\left\langle A^{i} A_{j}|v| A_{i} A^{j}\right\rangle_{\mathfrak{A}} \\
\bar{B}_{A_{i} \rightarrow A^{i}, A_{j} \rightarrow A^{j}} & =\left\langle A_{i} A_{j}|v| A^{i} A^{j}\right\rangle_{\mathfrak{A}} .
\end{aligned}
$$

Finally, $f_{A, B}$ designates matrix elements of the Fock operator $f_{A, B}=\langle A|f| B\rangle$ and $\langle A B|v| C D\rangle_{\mathfrak{A}}$ labels anti-symmetrized two-electron matrix elements $\langle A B|v| C D\rangle_{\mathfrak{A}}=$ $\langle A B|v| C D\rangle-\langle A B|v| D C\rangle$ in the Dirac notation.

The positive definiteness of $\delta^{(2)} E\left[\Phi_{0}\right]$ then requires all the eigenvalues $\lambda_{i}$ of the characteristic problem

$$
\left[\begin{array}{ll}
\mathbf{A} & \mathbf{B} \\
\overline{\mathbf{B}} & \overline{\mathbf{A}}
\end{array}\right]\left[\begin{array}{l}
\mathbf{D}_{\mathbf{i}} \\
\overline{\mathbf{D}}_{i}
\end{array}\right]=\lambda_{i}\left[\begin{array}{c}
\mathbf{D}_{\mathbf{i}} \\
\overline{\mathbf{D}}_{i}
\end{array}\right]
$$

to be positive. When $\mathbf{A}$ and $\mathbf{B}$ are real, this problem may be factorized into the two subproblems for matrices $(\mathbf{A}+\mathbf{B})$ and $(\mathbf{A}-\mathbf{B})$ of half the dimension. It can also be shown [23] that in the presence of the instability (or instabilities in the presence of several negative roots $\lambda_{i}$ ) the eigenvector associated with the lowest negative eigenvalue gives the direction of the steepest descent on the energy hypersurface $E[\Phi]$ at $\Phi=\Phi_{0}$. This fact can be conveniently exploited when searching for BS HF solutions [23].

\subsection{Spin-adapted stability conditions}

In molecular electronic structure investigations one generally employs a spin-free electronic Hamiltonian that commutes with both total spin operators $S^{2}$ and $S_{z}$ and one relies on simple MSOs given by a product of orbital and pure-spin parts, i.e., $|A\rangle=|a\rangle|\sigma\rangle$ with $\sigma=\alpha$ or $\beta$ (or $\pm \frac{1}{2}$ ). In the closed-shell case the MOs $|a\rangle$ are 
doubly occupied so that the relevant wave function represents a pure singlet state with $S_{z}=S=0$. One can thus formulate spin-symmetry restricted stability conditions $[19,23]$ by carrying out projections onto the singlet- and triplet-coupled manifolds of monoexcitations $A_{i} \rightarrow A^{i}$. This yields the so-called singlet and triplet (or nonsinglet) stability conditions $[23,29]$. The former ones preserve the zero total spin and in the presence of instability lead to closed-shell type BS solutions with doubly occupied MOs while the triplet instability implies the existence of spin-BS solutions, referred to as unrestricted HF (UHF) solutions of the DODS (i.e., different orbitals for different spins) type.

Distinguishing the corresponding $\mathbf{A}$ and $\mathbf{B}$ matrices and their matrix elements by the superscripts $s$ and $t$ for the singlet and triplet stability problem, respectively, we then obtain

$$
\begin{aligned}
& A_{a_{i} \rightarrow a^{i}, a_{j} \rightarrow a^{j}}^{s, t}=f_{a^{i}, a^{j}} \delta_{a_{j}, a_{i}}-f_{a_{j}, a_{i}} \delta_{a^{i}, a^{j}}+2 \tau\left\langle a^{i} a_{j}|v| a_{i} a^{j}\right\rangle-\left\langle a^{i} a_{j}|v| a^{j} a_{i}\right\rangle, \\
& B_{a_{i} \rightarrow a^{i}, a_{j} \rightarrow a^{j}}^{s, t}=2 \tau\left\langle a_{i} a_{j}|v| a^{i} a^{j}\right\rangle-\left\langle a_{i} a_{j}|v| a^{j} a^{i}\right\rangle,
\end{aligned}
$$

where $\tau=1$ in the singlet case and $\tau=0$ in the triplet case. Here we must note that more complex stability problems will be encountered if one relies on general MSOs given as a linear combination of spin-up and spin-down components (for details see $[18,21])$.

In this paper we shall require spin-preserving stability conditions for a simple openshell case, i.e., for the ROHF solutions. These so-called doublet stability conditions (recall that the ROHF solutions are always non-doublet unstable) that preserve the ROHF wave function form $\left|\Phi_{0}\right\rangle=\mathfrak{A}\left|a_{1}, \bar{a}_{1}, a_{2}, \bar{a}_{2}, \cdots, a_{n}, \bar{a}_{n}, a_{0}\right\rangle$, where an overbar indicates the down spin, take a more complex form since we have to distinguish different types of monoexcitations (namely, occupied to open-shell $a_{i} \rightarrow a_{0}$, occupied to virtual $a_{i} \rightarrow a^{i}$, and open-shell to virtual $a_{0} \rightarrow a^{i}$ excitations). We thus have $[27,28]$

$$
\begin{aligned}
A_{a_{0} \rightarrow a^{i}, a_{0} \rightarrow a^{j}}^{d}= & f_{a^{i}, a^{j}}-f_{a_{0}, a_{0}} \delta_{a^{i}, a^{j}} \\
A_{a_{0} \rightarrow a^{i}, a_{i} \rightarrow a}^{d}= & -f_{a_{i}, a_{0}} \delta_{a^{i}, a}+\left\langle a^{i} a_{i}|v| a_{0} a\right\rangle \\
A_{a_{i} \rightarrow a, a_{j} \rightarrow a^{\prime}}^{d}= & \delta_{a_{i}, a_{j}}\left(2 \delta_{a, a^{\prime}} f_{a_{0}, a_{0}}-\delta_{a, a_{0}} f_{a_{0}, a^{\prime}}\right)-2 \delta_{a, a^{\prime}} f_{a_{j}, a_{i}}+\delta_{a_{0}, a^{\prime}} \delta_{a, a_{0}} f_{a_{j}, a_{i}} \\
& +\delta_{a_{j}, a_{i}}\left(2 f_{a, a^{\prime}}-\delta_{a_{0}, a^{\prime}} f_{a, a_{0}}\right)+2\left\langle a_{j} a|v| a^{\prime} a_{i}\right\rangle_{\mathfrak{a}} \\
& +\delta_{a_{j}, a_{i}}\left\langle a a_{0}|v| a^{\prime} a_{0}\right\rangle_{\mathfrak{a}}-\delta_{a, a^{\prime}}\left\langle a_{j} a_{0}|v| a_{i} a_{0}\right\rangle_{\mathfrak{a}}-\delta_{a, a_{0}}\left\langle a_{j} a_{0}|v| a^{\prime} a_{i}\right\rangle_{\mathfrak{a}} \\
& -\delta_{a_{0}, a^{\prime}}\left\langle a_{j} a|v| a_{0} a_{i}\right\rangle_{\mathfrak{a}}-\left(2-\delta_{a, a_{0}}\right) \delta_{a, a^{\prime}} \delta_{a_{i}, a_{j}} f_{a_{0}, a_{0}},
\end{aligned}
$$

and

$$
\begin{aligned}
B_{a_{0} \rightarrow a^{i}, a_{0} \rightarrow a^{j}}^{d} & =0 \\
B_{a_{0} \rightarrow a^{i}, a_{i} \rightarrow a}^{d} & =-\delta_{a_{0}, a} f_{a_{i}, a^{i}}+\left\langle a_{i} a_{0}|v| a a^{i}\right\rangle_{\mathfrak{a}}=B_{a_{i} \rightarrow a, a_{0} \rightarrow a^{i}}^{d} \\
B_{a_{i} \rightarrow a, a_{j} \rightarrow a^{\prime}}^{d} & =2\left\langle a_{i} a_{j}|v| a a^{\prime}\right\rangle_{\mathfrak{a}}-\delta_{a_{0}, a}\left\langle a_{i} a_{j}|v| a_{0} a^{\prime}\right\rangle_{\mathfrak{a}}-\delta_{a_{0}, a^{\prime}}\left\langle a_{i} a_{j}|v| a a_{0}\right\rangle_{\mathfrak{a}},
\end{aligned}
$$

where non-indexed orbital labels $a$ and $a^{\prime}$ designate either the open-shell orbital $a_{0}$ or the virtual orbital $a^{k}$. The anti-symmetrized two-electron integrals are now defined as follows

$$
\langle a b|v| c d\rangle_{\mathfrak{a}} \equiv 2\langle a b|v| c d\rangle-\langle a b|v| d c\rangle
$$




\section{Model description}

We employ a simple $\pi$-electron model of the allyl radical as described by the PPP electronic Hamiltonian $H_{\mathrm{PPP}}$,

$$
H_{\mathrm{PPP}}=\sum_{i, j} z_{i j} E_{i j}+\frac{1}{2} \sum_{i, j} \gamma_{i j}\left(E_{i i} E_{j j}-\delta_{i j} E_{i i}\right)
$$

where $E_{i j}$ are the generators of the orbital unitary group $\mathrm{U}(n), E_{i j}=\sum_{\sigma} X_{i \sigma}^{\dagger} X_{j \sigma}$, with $X_{i \sigma}^{\dagger}$ and $X_{i \sigma}$ representing, respectively, the creation and annihilation operators defined on a hypothetical basis of symmetrically-orthonormalized set of $2 \mathrm{p}_{z}$ carbon atomic spin-orbitals $|i \sigma\rangle=|i\rangle|\sigma\rangle, \sigma= \pm \frac{1}{2}$. The one-particle matrix elements $z_{i j}$ have the form

$$
z_{i j}= \begin{cases}\alpha_{i}-\sum_{j(\neq i)} Z_{j} \gamma_{i j} & \text { if } i=j \\ \beta_{i j} & \text { if } i, j \text { are nearest neighbors } \\ 0 & \text { otherwise }\end{cases}
$$

where $\alpha_{i}$ is the so-called Coulomb integral and $Z_{i}$ designates the number of $\pi$-electrons contributed by the $i$-th atomic site. For off-diagonal one-body matrix elements $z_{i j}$ one generally invokes the tight-binding approximation so that only resonance integrals (or hopping terms) $\beta_{i j}$ between the nearest-neighbor sites $i$ and $j$ are accounted for. The two-body terms $\gamma_{i j}$ then represent two-electron Coulomb integrals $\gamma_{i j}=\langle i j|v| i j\rangle$. All other two-electron integrals are neglected in view of the zero-differential overlap approximation. In the case of neutral, unsaturated hydrocarbons when $Z_{i}=1$ the above Hamiltonian is often written in the form (see, e.g., Eq. (6.19) of [47])

$$
H_{\mathrm{PPP}}^{\prime}=H_{\mathrm{PPP}}+H_{\mathrm{nucl}}=\sum_{i, j}^{\prime} \beta_{i j} E_{i j}+\frac{1}{2} \sum_{i, j} \gamma_{i j}\left(E_{i i}-1\right)\left(E_{j j}-1\right),
$$

where we assumed all one-center integrals to be equivalent so that $\alpha_{i}=\alpha \equiv 0$ and $\gamma_{i i}=\gamma_{11}$ and where we added the nuclear repulsion energy $H_{\text {nucl }}$ as given by the Goeppert-Mayer and Sklar approximation [48], $H_{\text {nucl }}=\sum_{i j} Z_{i} Z_{j} \gamma_{i j}$. The prime on the first summation symbol implies that only nearest-neighbor resonance integrals are to be accounted for.

For the studied $\pi$-electron model of the allyl radical we assume the three carbon centers to form the vertices of a triangle with a fixed $\widehat{C C C}$ angle of $2 \pi / 3$ and, generally, different $\mathrm{C}-\mathrm{C}$ bond-lengths $d_{1}$ and $d_{2}$ (cf. Fig. 1a). For non-symmetric structures with $d_{1} \neq d_{2}$ we assume that $d_{1}+d_{2}=2 d$, representing a cut through the $\pi$-electronic PES that is perpendicular to the line $d_{1}=d_{2}$ characterizing symmetric structures (cf., e.g., [5]). The $\pi$-electron distribution for the symmetric and asymmetric geometries is then schematically indicated in Fig. 1b. For symmetric structures the SA solution occurs always at $\omega=\pi / 4$. In the fully correlated limit $(\beta=0)$ the $(\mathrm{BS})$ solutions occur at $\omega=0$ and $\pi / 2$. The diagrams in Fig. $1 \mathrm{~b}$ may also be regarded as the valence bond (VB) structures related by blue double arrows, the red double arrows implying the relationship of stable solutions for distorted structures with BS ones for symmetric structures. For a symmetric system with equal bond-lengths, $d_{1}=d_{2}=d$, we choose the standard C-C bond length $d=1.4 \AA$ and we also set $\beta_{\mu \nu}=\beta$ in which case the only semi-empirical parameters are the resonance integral $\beta$ (whose 
(a)

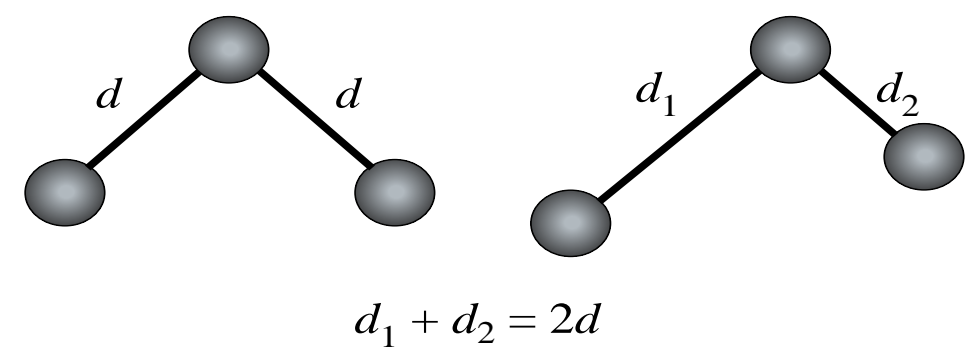

(b)

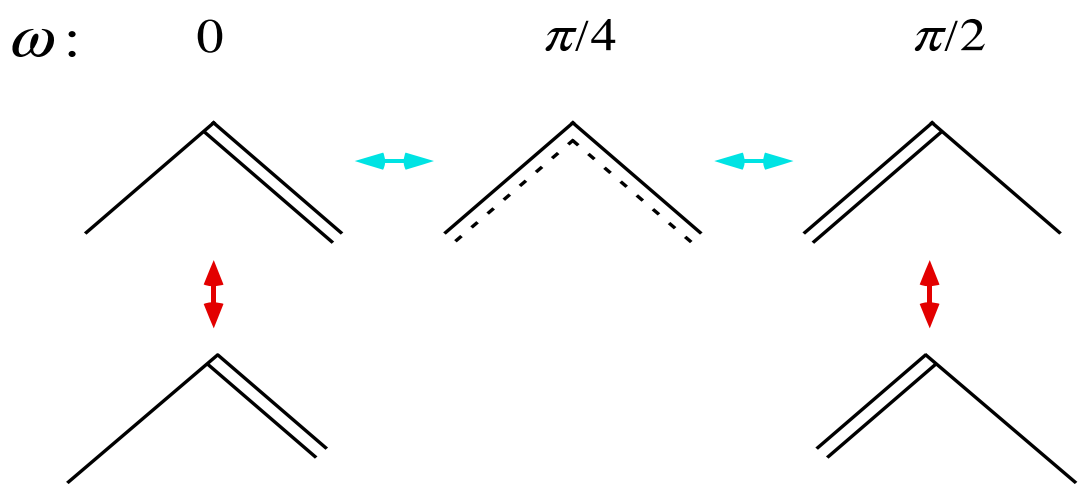

Figure 1: Schematic representation of the studied $\pi$-electron model of the allyl radical. (a) Structure of the carbon nuclear framework for the symmetric and distorted, broken-symmetry situations. (b) Schematic $\pi$-electron distribution in the symmetryadapted (SA) and broken-symmetry (BS) solutions.

spectroscopic value is usually set equal to $-2.4 \mathrm{eV}$ ) and the two-electron Coulomb integrals $\gamma_{\mu \nu}$ that are evaluated using various approximations (see, e.g., [49]). For the sake of simplicity we employ a modified, simple point-charge approximation so as to obtain a finite on-site self-interaction for $\gamma_{\mu \mu} \equiv \gamma_{11}$ which is referred to as the Mataga-Nishimoto approximation [50], i.e.,

$$
\gamma_{\mu \nu}=e^{2} /\left(R_{\mu \nu}+a\right),
$$

where $R_{\mu \nu}$ is the distance between the centers $\mu$ and $\nu$. For the one-center integral $\gamma_{11}$ one then employs the so-called $(I-A)$ approximation [48] with $I$ and $A$ designating, respectively, the $2 \mathrm{p}_{z}$ atomic orbital, valence state ionization potential and electron affinity, $\gamma_{11}=I-A$. The generally accepted value for $\gamma_{11}$ is $\gamma_{11}=e^{2} / a=10.84 \mathrm{eV}$, which in turn determines the parameter $a$ in Eq. (14).

We note in passing that by considering only the on-site integrals $\gamma_{11} \equiv \gamma_{0}$ by setting $\gamma_{\mu \nu}=\gamma_{0} \delta_{\mu \nu}$ we obtain the Hubbard Hamiltonian in which case the hopping integral $\beta$ is usually designated as $\beta \equiv-t$ and the on-site Coulomb integral as $\gamma_{0} \equiv U$. This makes it possible to explore the entire correlation range in terms of a single parameter $U / t$ representing the so-called coupling constant. Although the PPP Hamiltonian handles the electron repulsion in a more realistic way (which is essential 
for spectroscopic purposes, see, e.g. [51]) we can still employ the inverse value of the resonance integral $\beta$ as a coupling constant while keeping the Coulomb integrals fixed at their values given by Eq. (14) and thus explore the entire correlation regime. This is straightforward not only when we can regard the $\mathrm{C}-\mathrm{C}$ bonds as equivalent, so that $\beta_{\mu \nu}=\beta$ with $\mu$ and $\nu$ being nearest neighbors, but also when different resonance integrals must be employed as in the case of asymmetric, distorted geometries when we explore the entire PES. We then associate a fixed value of $\beta$ with a standard $\mathrm{C}-\mathrm{C}$ bond $\left(R_{e}=1.4 \AA\right)$ and evaluate the resonance integrals $\beta(R)$ for stretched bonds with bond-length $R$ by relying on the so-called Mulliken "magic" formula (see, e.g., [5]) which assumes proportionality of the resonance integrals to the corresponding overlap integrals $S(R)$, i.e.,

$$
\frac{\beta(R)}{\beta\left(R_{e}\right)}=\frac{S(R)}{S\left(R_{e}\right)}
$$

For $2 \mathrm{p}_{z}$ carbon atomic orbitals in $\pi$-orientation which are $R(\AA)$ apart we have then

$$
S(R)=\frac{1}{15} \exp (-\rho)\left[\rho^{3}+6 \rho^{2}+15 \rho+15\right], \quad \rho=\xi R / a_{0},
$$

where $\xi=1.625$ and $a_{0}$ is the Bohr radius $\left(a_{0}=0.52917 \AA\right)$. We note that when $\beta \rightarrow 0$ we reach the fully correlated limit while $\beta \rightarrow \infty$ (for practical purposes $\beta \approx 5$ $\mathrm{eV}$ ) corresponds to the uncorrelated (Hückel) limit.

\section{Stability conditions for the symmetric model}

We first apply the doublet stability conditions of Sec. 3.2 to a standard symmetric case having equivalent $\mathrm{C}-\mathrm{C}$ bonds. Numbering sequentially carbon atomic sites in Fig. 1a we designate one- and two-electron atomic integrals as follows: $\beta_{12}=\beta_{23} \equiv \beta$ and $\gamma_{11} \equiv \gamma_{0}, \gamma_{12}=\gamma_{23} \equiv \gamma_{1}, \gamma_{13} \equiv \gamma_{2}$, respectively. The LCAO MOs (labeled as $|1\rangle$, $|0\rangle$, and $|-1\rangle$ ) are in this case fully determined by the symmetry of the model, so that

$$
\left[\begin{array}{l}
|1\rangle \\
|0\rangle \\
|-1\rangle
\end{array}\right]=\frac{1}{2}\left[\begin{array}{ccc}
1 & \sqrt{2} & 1 \\
\sqrt{2} & 0 & -\sqrt{2} \\
1 & -\sqrt{2} & 1
\end{array}\right]\left[\begin{array}{l}
\left|\chi_{1}\right\rangle \\
\left|\chi_{2}\right\rangle \\
\left|\chi_{3}\right\rangle
\end{array}\right],
$$

where $\left|\chi_{i}\right\rangle$ designate the effective orthonormal AOs of the PPP model. In the following we specify the MOs by their labels 1,0 , and -1 , so that the ground state ROHF wave function may be simply written as $\left|\Phi_{0}\right\rangle=\mathfrak{A}|1 \overline{1} 0\rangle$, the bar indicating again the down spin.

We can now easily evaluate the one- and two-electron integrals over the MOs, Eq. (17), recalling that the one-electron matrix $\mathbf{z}=\left[z_{\mu \nu}\right]$ has the form

$$
\mathbf{z}=\left[\begin{array}{ccc}
-\left(\gamma_{1}+\gamma_{2}\right) & \beta & 0 \\
\beta & -2 \gamma_{1} & \beta \\
0 & \beta & -\left(\gamma_{1}+\gamma_{2}\right)
\end{array}\right]
$$

We next evaluate the relevant matrix elements of the doublet stability problem, 
Eqs. (8) and (9), obtaining

$$
\begin{aligned}
A_{1 \rightarrow 0,1 \rightarrow 0}^{\mathrm{d}} & =-\sqrt{2} \beta+\left(\gamma_{0}-\gamma_{2}\right) / 8=A_{0 \rightarrow-1,0 \rightarrow-1}^{\mathrm{d}}, \\
A_{1 \rightarrow 0,0 \rightarrow-1}^{\mathrm{d}} & =\left(\gamma_{0}-\gamma_{2}\right) / 4, \\
A_{1 \rightarrow 0,1 \rightarrow-1}^{\mathrm{d}} & =0=A_{0 \rightarrow-1,1 \rightarrow-1}^{\mathrm{d}}, \\
A_{1 \rightarrow-1,1 \rightarrow-1}^{\mathrm{d}} & =-4 \sqrt{2} \beta-\left(\gamma_{0}-\gamma_{2}\right) / 8+\left(\gamma_{0}+\gamma_{1}\right) / 2,
\end{aligned}
$$

and

$$
\begin{aligned}
B_{1 \rightarrow 0,1 \rightarrow 0}^{\mathrm{d}} & =B_{1 \rightarrow 0,1 \rightarrow-1}^{\mathrm{d}}=B_{0 \rightarrow-1,0 \rightarrow-1}^{\mathrm{d}}=B_{0 \rightarrow-1,1 \rightarrow-1}^{\mathrm{d}}=0, \\
B_{1 \rightarrow 0,0 \rightarrow-1}^{\mathrm{d}} & =B_{0 \rightarrow-1,1 \rightarrow 0}^{\mathrm{d}}=3\left(\gamma_{0}-\gamma_{2}\right) / 8, \\
B_{1 \rightarrow-1,1 \rightarrow-1}^{\mathrm{d}} & =\gamma_{0}-\gamma_{1}-\left(\gamma_{0}-\gamma_{2}\right) / 4 .
\end{aligned}
$$

The $3 \times 3$ stability matrices $\mathbf{A}^{\mathrm{d}} \pm \mathbf{B}^{\mathrm{d}}$ factorize into a $2 \times 2$ and $1 \times 1$ blocks since the doubly occupied MO $|1\rangle$ and the virtual MO $|-1\rangle$ are symmetric while the singly occupied MO $|0\rangle$ is antisymmetric with respect to the interchange $\left|\chi_{1}\right\rangle \rightleftarrows\left|\chi_{3}\right\rangle$, so that $\left(A^{\mathrm{d}} \pm B^{\mathrm{d}}\right)_{1 \rightarrow 0,1 \rightarrow-1}=\left(A^{\mathrm{d}} \pm B^{\mathrm{d}}\right)_{0 \rightarrow-1,1 \rightarrow-1}=0$. The relevant $2 \times 2$ stability block that yields the lowest lying root is then

$$
\mathbf{A}^{\mathrm{d}}+\mathbf{B}^{\mathrm{d}}=\left[\begin{array}{cc}
\kappa-\lambda & 5\left(\gamma_{0}-\gamma_{2}\right) / 8 \\
5\left(\gamma_{0}-\gamma_{2}\right) / 8 & \kappa-\lambda
\end{array}\right], \quad \kappa=-\sqrt{2} \beta+\left(\gamma_{0}-\gamma_{2}\right) / 8
$$

so that

$$
\lambda_{ \pm}=\kappa \pm 5\left(\gamma_{0}-\gamma_{2}\right) / 8=-\sqrt{2} \beta+\left(\gamma_{0}-\gamma_{2}\right) / 8 \pm 5\left(\gamma_{0}-\gamma_{2}\right) / 8
$$

Thus the lowest root $\lambda_{-}$,

$$
\lambda_{-}=-\sqrt{2} \beta-\left(\gamma_{0}-\gamma_{2}\right) / 2,
$$

leads to the stability condition

$$
|\beta|>\left(\gamma_{0}-\gamma_{2}\right) /(2 \sqrt{2}) \approx 2.48 \mathrm{eV}
$$

We thus see that the ROHF instability onset occurs in the vicinity of the spectroscopic value for the resonance integral. This agrees well with an earlier $a b$ initio result $[1,2]$ for the allyl radical where only a minimal symmetric stretch of the $\mathrm{C}-\mathrm{C}$ bonds was required in order to reach the instability range of geometries.

\section{Direct analytical approach}

In view of the simplicity of our model we can explore the stability of its ROHF solutions directly since the appropriate variational space for the mean energy functional $E[\Phi]$ is one-dimensional and can thus be parametrized by a single parameter designated below by $\omega$ (for more general parametrizations which allow breaking of other than spatial symmetries, see [52]). We thus generalize the MOs of Eq. (17) via $\omega$-dependent orthonormal MOs

$$
\left[\begin{array}{l}
|1\rangle \\
|0\rangle \\
|-1\rangle
\end{array}\right]=\frac{1}{\sqrt{2}}\left[\begin{array}{ccc}
s & 1 & c \\
\sqrt{2} c & 0 & -\sqrt{2} s \\
s & -1 & c
\end{array}\right]\left[\begin{array}{l}
\left|\chi_{1}\right\rangle \\
\left|\chi_{2}\right\rangle \\
\left|\chi_{3}\right\rangle
\end{array}\right],
$$


where

$$
c \equiv \cos \omega \quad \text { and } \quad s \equiv \sin \omega .
$$

In this way we can cover the entire region of $\mathrm{BS}$ solutions with $\omega=\pi / 4$ representing the symmetric MOs (17) while $\omega=0$ and $\omega=\pi / 2$ yield fully localized orbitals in one of the $\mathrm{C}-\mathrm{C}$ bonds (cf. Fig. 1b). Note that here we have exploited the alternancy symmetry of our PPP Hamiltonian (see, e.g., [53]) which implies that the MOs $|1\rangle$ and $|-1\rangle$ form an alternantly-conjugate pair while the MO $|0\rangle$ is self-conjugate. Moreover, the AOs are orthonormal by definition so that the matrix relating the MOs with the atomic ones must be orthogonal.

Clearly, the MOs (25) also describe asymmetrically distorted models with unequal $\mathrm{C}-\mathrm{C}$ bonds (see the bottom part of Fig. 1b). For this reason we will thus distinguish the resonance and two-electron Coulomb integrals for distorted structures by relying on the following shorthand notation

$$
\begin{aligned}
\beta & \equiv \beta_{12}, \quad \beta^{\prime} \equiv \beta_{23} \\
\gamma_{0} & \equiv \gamma_{11}, \quad \gamma_{1} \equiv \gamma_{12}, \quad \gamma_{1}^{\prime} \equiv \gamma_{23}, \quad \gamma_{2} \equiv \gamma_{13},
\end{aligned}
$$

so that the $\mathbf{z}$ matrix takes the form

$$
\mathbf{z}=\left[\begin{array}{ccc}
-\left(\gamma_{1}+\gamma_{2}\right) & \beta & 0 \\
\beta & -\left(\gamma_{1}+\gamma_{1}^{\prime}\right) & \beta^{\prime} \\
0 & \beta^{\prime} & -\left(\gamma_{1}^{\prime}+\gamma_{2}\right)
\end{array}\right]
$$

The total energy $E(\omega)$ that is associated with the wave function $|\Phi(\omega)\rangle=\mathfrak{A}|1 \overline{1} 0\rangle$ is given by

$$
E(\omega)=2\langle 1|z| 1\rangle+\langle 0|z| 0\rangle+\langle 11|v| 11\rangle+\langle 10|v| 10\rangle_{\mathfrak{a}} .
$$

The required integrals are easily evaluated (see Appendix A) yielding the following explicit expression for the energy

$$
E(\omega)=2\left(\beta s+\beta^{\prime} c\right)+\frac{1}{2} \gamma_{0}\left(1+s^{2} c^{2}\right)-\gamma_{1}\left(1+\frac{1}{2} s^{2}\right)-\gamma_{1}^{\prime}\left(1+\frac{1}{2} c^{2}\right)-\gamma_{2}\left(1+\frac{1}{2} s^{2} c^{2}\right) .
$$

For the symmetric case $(\omega=\pi / 4)$ we thus obtain the energy

$$
E(\pi / 4)=\sqrt{2} \beta+\frac{5}{8} \gamma_{0}-\frac{5}{2} \gamma_{1}-\frac{9}{8} \gamma_{2}
$$

and, in general, identifying primed and unprimed parameters (e.g., setting $\beta=\beta^{\prime}$ and $\gamma_{1}=\gamma_{1}^{\prime}$ ) we get

$$
E_{\mathrm{sym}}(\omega) \equiv \mathcal{E}(\omega)=2 \beta(s+c)+\frac{1}{2} \gamma_{0}\left(1+s^{2} c^{2}\right)-\frac{5}{2} \gamma_{1}-\gamma_{2}\left(1+\frac{1}{2} s^{2} c^{2}\right) .
$$

Differentiating with respect to $\omega$ we get

$$
\begin{aligned}
\mathcal{E}^{\prime}(\omega) & =2 \beta(c-s)+\frac{1}{4}\left(\gamma_{0}-\gamma_{2}\right) \sin (4 \omega) \\
& =(c-s)\left[2 \beta+\left(\gamma_{0}-\gamma_{2}\right) \operatorname{sc}(c+s)\right]
\end{aligned}
$$

so that $\mathcal{E}^{\prime}(\pi / 4)=0$ for whichever parametrization is employed, as expected. 
In order to derive the stability condition for this ROHF solution we calculate the second derivative, obtaining

$$
\mathcal{E}^{\prime \prime}(\omega)=-2 \beta(s+c)+\left(\gamma_{0}-\gamma_{2}\right) \cos (4 \omega)
$$

so that for the stability of our ROHF wave function we require that

$$
\mathcal{E}^{\prime \prime}(\pi / 4)=-2 \sqrt{2} \beta-\left(\gamma_{0}-\gamma_{2}\right)>0,
$$

which is identical to the condition (24) derived earlier by relying on general stability conditions for a simple open-shell case, Sec. 5. We note that for $\omega=0$ or $\pi / 2$ we have $\mathcal{E}^{\prime}(0)=-\mathcal{E}^{\prime}(\pi / 2)=2 \beta$, so that for $\beta=0$ we have again a HF solution (i.e., a stationary point) with $\mathcal{E}^{\prime \prime}(0)=\mathcal{E}^{\prime \prime}(\pi / 2)=\gamma_{0}-\gamma_{2}>0$, implying stable BS HF solutions.

In general, for a fixed geometry the symmetry-adapted HF solutions are unstable for the coupling constants - given as the reciprocal value of the resonance integral $\beta$ - in the interval $0 \geq \beta>\beta_{\text {crit }}$, where $\beta_{\text {crit }}=-\left(\gamma_{0}-\gamma_{2}\right) /(2 \sqrt{2})$. To each such $\beta$ value corresponds an $\omega$ value $\omega_{\text {opt }}$ yielding a BS HF solution given by the vanishing of the square bracket in the expression (33) for the first derivative of the energy mean value, namely

$$
\beta=-\frac{1}{2}\left(\gamma_{0}-\gamma_{2}\right) f(\omega) \quad \text { with } \quad f(\omega) \equiv f=s c(c+s) .
$$

Note that $f(\omega)$ is symmetric about $\omega=\pi / 4$, i.e., $f(\omega)=f(\pi / 2-\omega)$, where it reaches its maximum value $f(\pi / 4)=1 / \sqrt{2}$, so that we always obtain two degenerate BS solutions related via the reflection in the plane passing through the central $\mathrm{C}_{2}$ atom and perpendicular to the molecular plane. Thus, $1<f<1 / \sqrt{2}$ for $0<\omega<\pi / 2$, so that a $\mathrm{BS} \mathrm{HF}$ solution exists for all $\beta$ values in the interval $0 \geq \beta>\beta_{\text {crit }}$ as also implied by the plot of $\beta$ vs. $\omega_{\text {opt }}$ shown in Fig. 2 .

The inverse relationship to (36), i.e., $\omega_{\text {opt }}=f^{-1}\left(\beta_{\text {opt }} / K\right) ; K=-\frac{1}{2}\left(\gamma_{0}-\gamma_{2}\right)$ is clearly multiply (i.e., doubly) valued, each branch corresponding to one degenerate $\mathrm{BS}$ solution. For each $\beta$ in the interval $0 \geq \beta>\beta_{\text {crit }}, \quad \beta_{\text {crit }}=-\left(\gamma_{0}-\gamma_{2}\right) /(2 \sqrt{2})$, we find two values of $\omega_{\text {opt }}$ yielding the corresponding BS ROHF solutions. The relationship (36) thus defines pairs $\left(\beta_{\text {opt }}, \omega_{\text {opt }}\right) \equiv\left(\beta\left(\omega_{\text {opt }}\right), \omega_{\text {opt }}\right)$ of $\beta$ and $\omega$ values which characterize stable, degenerate BS HF solutions given by the minima on the mean energy functional $E_{\text {sym }}(\omega)$ or $\mathcal{E}(\omega)$ curves for $0 \leq|\beta|<\left|\beta_{\text {crit }}\right|$ as shown in Fig. 3 . The stable, doubly degenerate BS ROHF solutions for $|\beta|<\left|\beta_{\text {crit }}\right|$ correspond to local minima and the unstable one to a maximum which turns into a minimum once $|\beta|>\left|\beta_{\text {crit }}\right|$, in which case only a stable symmetry-adapted ROHF solution exists. The entire mean energy variational (hyper-)surface $E$ as a function of $\beta$ and $\omega$ is illustrated in Fig. 4 . The cut (red curve online) at $\beta=\beta_{\text {crit }} \approx-2.48$ separates regions of stable and unstable ROHF solutions for $\omega=\pi / 4$. At this $\beta$ value the minima for $\beta<\beta_{\text {crit }}$ turn into maxima for $\beta>\beta_{\text {crit }}$ at $\omega=\pi / 4$.

Another clear indication of a singular behavior of the HF solutions at $\beta=\beta_{\text {crit }}$ is provided by the first-order density (bond-order) matrix $\left\|p_{\mu \nu}\right\|$. For the considered case of symmetric geometries we have that

$$
p_{12} \equiv s=\sin \omega, \quad p_{23} \equiv c=\cos \omega, \quad p_{13}=0, \quad p_{\mu \mu}=1, \mu=1,2,3,
$$




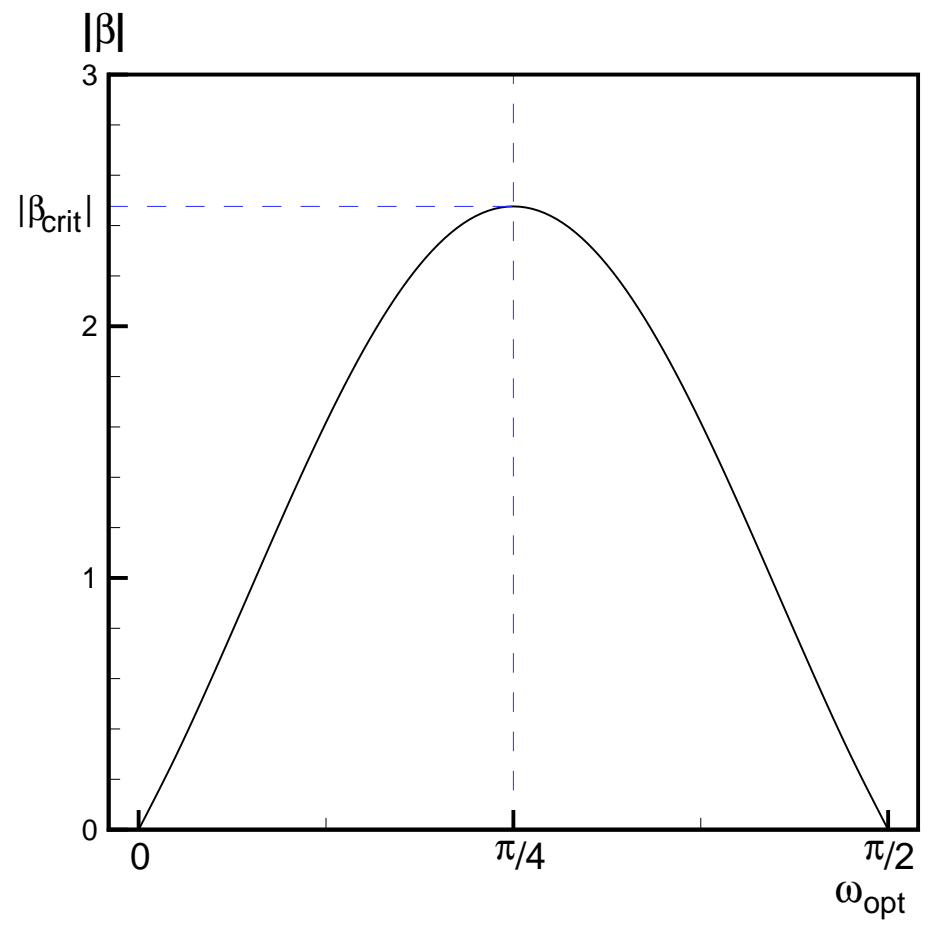

Figure 2: Dependence of the optimal values $\omega_{\text {opt }}$ of the variation parameter $\omega$ defining the BS ROHF solution vs. the corresponding resonance integral $\beta$.

as implied by the MOs (25) and (26). Thus, for $|\beta| \geq\left|\beta_{\text {crit }}\right|$, where the symmetric solutions are stable, we have that

$$
p_{12}=p_{23}=1 / \sqrt{2}, \quad p_{13}=0, \quad p_{\mu \mu}=1, \mu=1,2,3,
$$

while for $|\beta| \leq\left|\beta_{\text {crit }}\right|$ the bond orders $p_{12}$ and $p_{23}$ bifurcate as shown in Fig. 5 for the BS solutions with $\pi / 4 \leq \omega \leq \pi / 2$. For $0 \leq \omega \leq \pi / 4$ the role of $p_{12}$ and $p_{23}$ is interchanged (cf. also Fig. 2 of [26] for an analogous result in the closed-shell case of benzene).

\section{Breaking of a nuclear framework symmetry}

As already pointed out above the presence of a spin-preserving instability of a symmetry-adapted RHF or ROHF solution implies the existence of degenerate BS solutions of the first kind. This in turn implies the "instability" of the system towards a de facto symmetry breaking or distortion of the system as described by a Hamiltonian that is no longer invariant to symmetry operation(s) inter-relating degenerate BS solutions and yields HF solutions of the second kind. In the molecular case this entails an appropriate distortion of the nuclear framework along some symmetry-breaking coordinate (designated in the following by $\Delta$ or $\gamma$ ) characterizing such asymmetric geometries. It is thus important to distinguish BS solutions that are associated with a fully symmetric Hamiltonian or nuclear framework geometry (BS solutions of the 


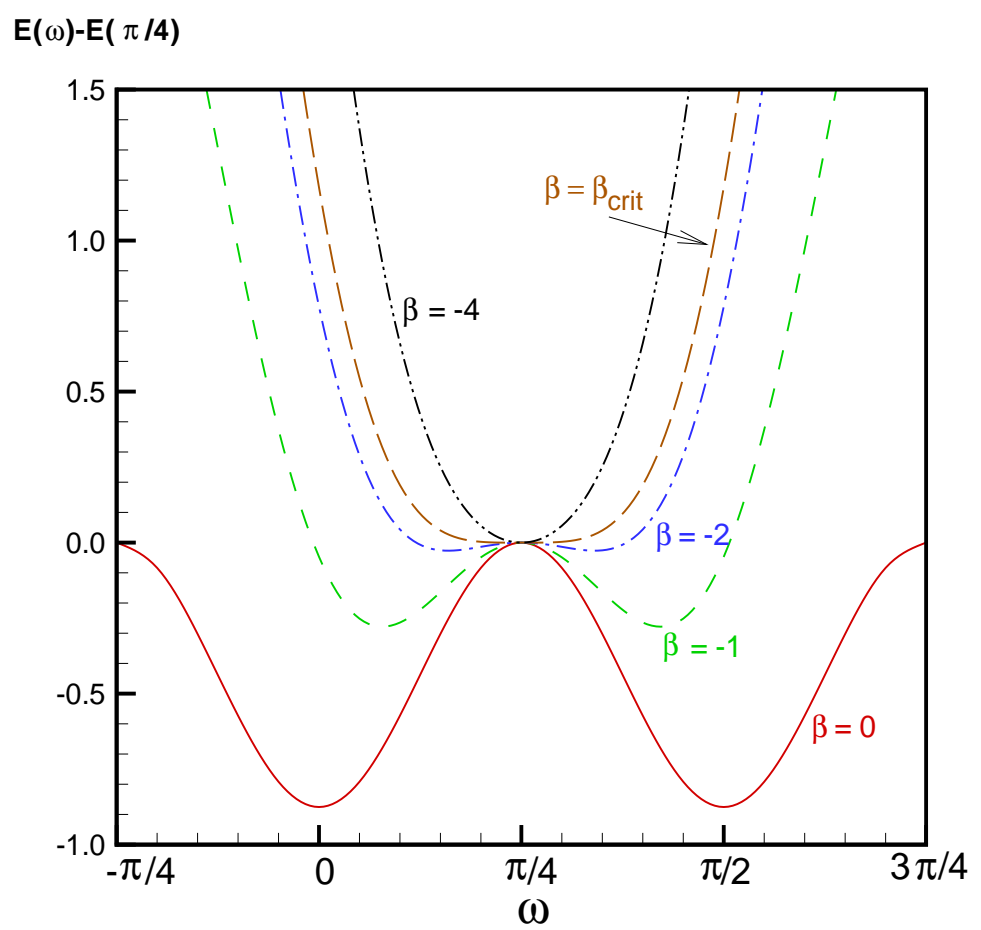

Figure 3: Mean energy value functional $E_{\text {sym }}(\omega)$, Eq. (32), dependence on the variational parameter $\omega$ for several values of the resonance integral $\beta(\beta=$ $0,-1,-1.5, \beta_{\text {crit }} \approx-2.48$, and $-3 \mathrm{eV}$ ). These plots are shifted to coincide at $\omega=\pi / 4$.

first kind) from those that are based on a correspondingly distorted geometry (BS solutions of the second kind). This distinction is not always sufficiently clear in the literature but it should be clear in the following from the context.

In the case of our simple model we can define $\Delta$ as the difference between the $\mathrm{C}_{1}-\mathrm{C}_{2}$ and $\mathrm{C}_{2}-\mathrm{C}_{3}$ bond lengths, i.e., $\Delta=d_{1}-d_{2}$, with $\Delta=0$ characterizing the undistorted symmetric structure (cf. Fig. 1a). Considering the bond lengths $d_{1}$ and $d_{2}$ as independent distortion (or vibrational) coordinates we can best explore the relevant PES by considering a PEC cut along the line $d_{1}+d_{2}=2 d$ for $d$ designating the standard $\mathrm{C}-\mathrm{C}$ bond length $d=1.4 \AA$. We can equivalently characterize the distorted structures by the ratio of the $d_{1}$ and $d_{2}$ bond lengths, namely by the parameter $\gamma=d_{1} / d_{2}$ that is simply related to $\Delta$, i.e.,

$$
\begin{aligned}
\Delta & =d_{1}-d_{2}=2 d \frac{\gamma-1}{\gamma+1}, \\
\gamma & =\frac{d_{1}}{d_{2}}=\frac{2 d+\Delta}{2 d-\Delta},
\end{aligned}
$$

where we assume that $d_{1}+d_{2}=2 d$.

Such a distortion of the nuclear framework will resolve the degeneracy of BS solutions at $\Delta=0$ or $\gamma=1$, yielding two solutions for the distorted structure, one 


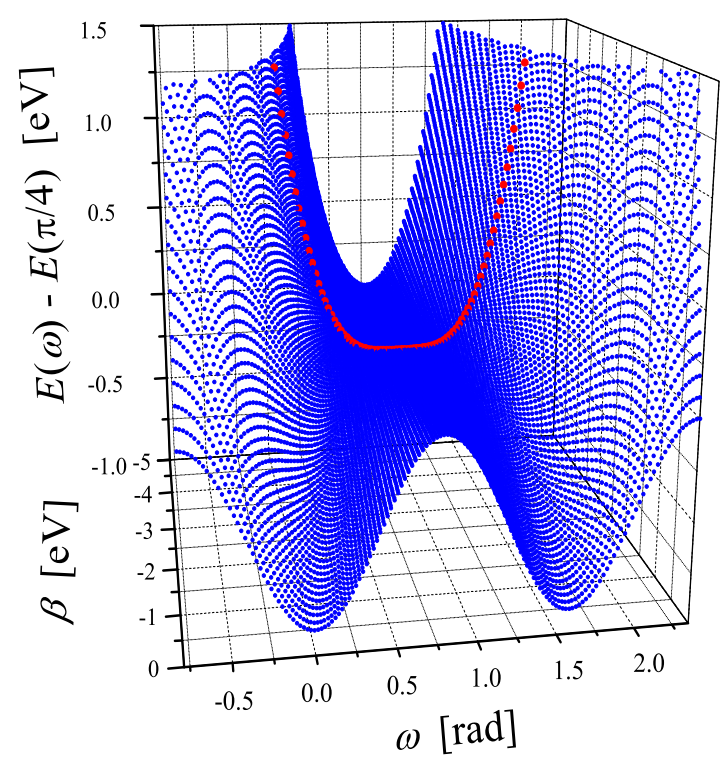

Figure 4: A 3D plot of the variational mean energy (hyper)surface $E(\beta, \omega)$ as a function of both the resonance integral $\beta$ and the variational parameter $\omega$ relative to the energy associated with the symmetric MOs defined by $\omega=\pi / 4 \approx 0.785 \mathrm{rad}$, c.f., Eqn. (31)

having a lower and the other one a higher energy than the degenerate BS solutions depending on whether the distortion is "in" or "out" of phase with the CDW of the $\Delta=0$ BS solution, respectively. This is illustrated in Fig. 6 where we plot the mean energy value functional $E_{\text {tot }}(\omega)$, Eq. (41), as a function of the variational parameter $\omega$ for several asymmetric structures characterized by the distortion parameter $\gamma$, Eq. (40), including that for the corresponding symmetric structure $(\gamma=1)$ for the resonance integral value $\beta=-1.5 \mathrm{eV}$. The stable ROHF solutions correspond to the local minima while the unstable solution is associated with the midlocated maximum. Note that an "in-phase" distortion lowers the energy of the $\gamma=1$ degenerate BS solution while the "out-of-phase" distortion raises this energy. For sufficiently large distortions the higher-lying minimum merges with the intermediate maximum into an inflexion point at $\gamma=\gamma_{\text {crit }}$ or $\Delta=\Delta_{\text {crit }}$. It is not difficult to see that, in general, the two resulting PECs will cross (with a finite angle of crossing) at the symmetric geometry $\Delta=0$ (for a schematic representation, see, e.g., Fig. 6 of [19] or Fig. 1(c) of [17]). However, a detailed shape of the entire PEC or PES, particularly in the neighborhood of the crossing, is not immediately apparent and difficult to compute in general situations.

In the studied simple example we can easily find all the HF solutions (both stable 


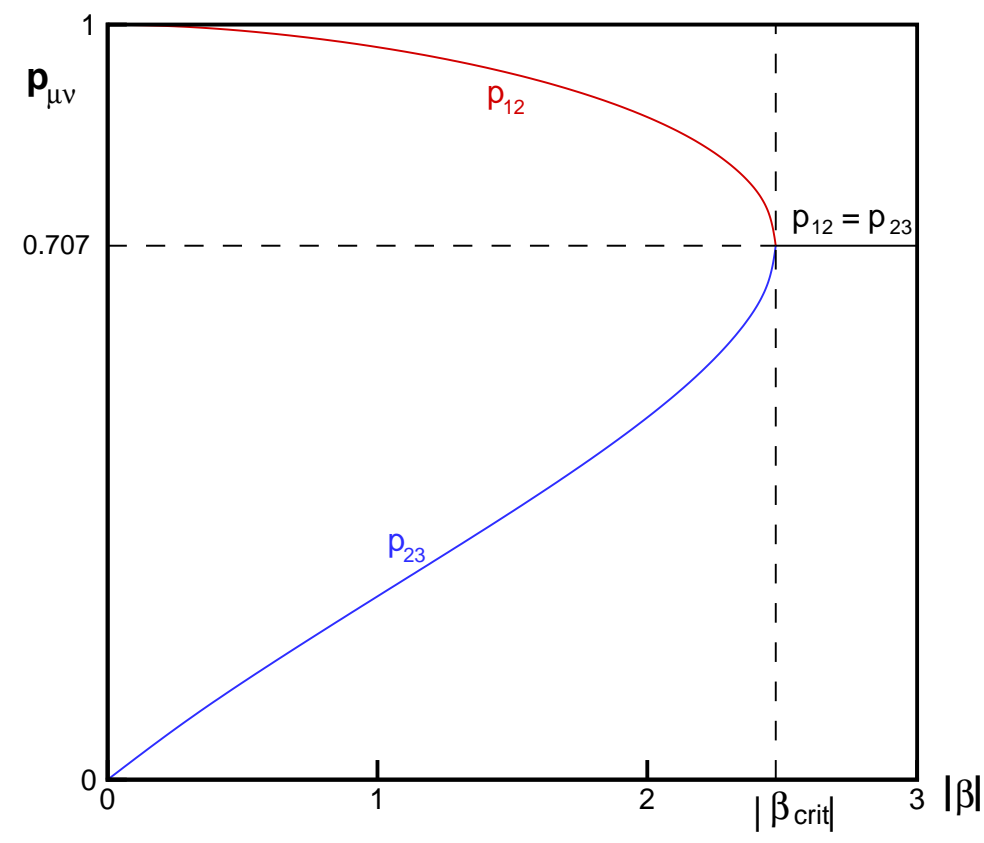

Figure 5: Plot of the first-order density (bond order) matrix off-diagonal elements $p_{12}$ and $p_{23}$ for the $\mathrm{SA}\left(|\beta| \geq\left|\beta_{\text {crit }}\right|\right)$ and $\mathrm{BS}\left(|\beta| \leq\left|\beta_{\text {crit }}\right|\right)$ HF solutions as a function of the resonance integral $\beta\left(\beta_{\text {crit }}=-2.48 \mathrm{eV}\right)$.

and unstable ones) for any geometry since our variational space is one dimensional. For this purpose it is useful to rewrite the total energy expression of Eq. (30) by adding the nuclear energy (in the Goeppert-Mayer and Sklar sense) $E_{\text {nucl }}=\gamma_{1}+\gamma_{1}^{\prime}+\gamma_{2}$ and define the total energy as follows

$$
E_{\text {tot }}(\omega) \equiv E(\omega)+E_{\text {nucl }}-\frac{1}{2} \gamma_{0}=2\left(\beta s+\beta^{\prime} c\right)+\frac{1}{8}\left(\gamma_{0}-\gamma_{2}\right) \sin ^{2}(2 \omega)-\frac{1}{2}\left(\gamma_{1} s^{2}+\gamma_{1}^{\prime} c^{2}\right) .
$$

We then have

$$
E_{\mathrm{tot}}^{\prime}(\omega)=2\left(\beta c-\beta^{\prime} s\right)+\frac{1}{4}\left(\gamma_{0}-\gamma_{2}\right) \sin (4 \omega)-\frac{1}{2}\left(\gamma_{1}-\gamma_{1}^{\prime}\right) \sin (2 \omega)
$$

and

$$
E_{\text {tot }}^{\prime \prime}(\omega)=2\left(\beta s+\beta^{\prime} c\right)+\left(\gamma_{0}-\gamma_{2}\right) \cos (4 \omega)-\left(\gamma_{1}-\gamma_{1}^{\prime}\right) \cos (2 \omega) .
$$

Using the above expressions we can now easily compute ROHF energies for any $\beta$ and $\gamma$ (or $\Delta$ ) values. We are particularly interested in the range of a coupling constant that yields unstable symmetry-adapted ROHF solutions in which case we can generate three solutions for each distorted structure with $\gamma \neq 1$ (or $\Delta \neq 0$ ), namely two stable ones associated with the minima on the $E_{\text {tot }}(\omega)$ curve and an unstable one associated with a maximum as is apparent from the plots of $E_{\text {tot }}(\omega)$ on $\omega$ in Fig. 6 . We simply have to find the respective $\omega_{\text {opt }}$ values given by the roots in the 


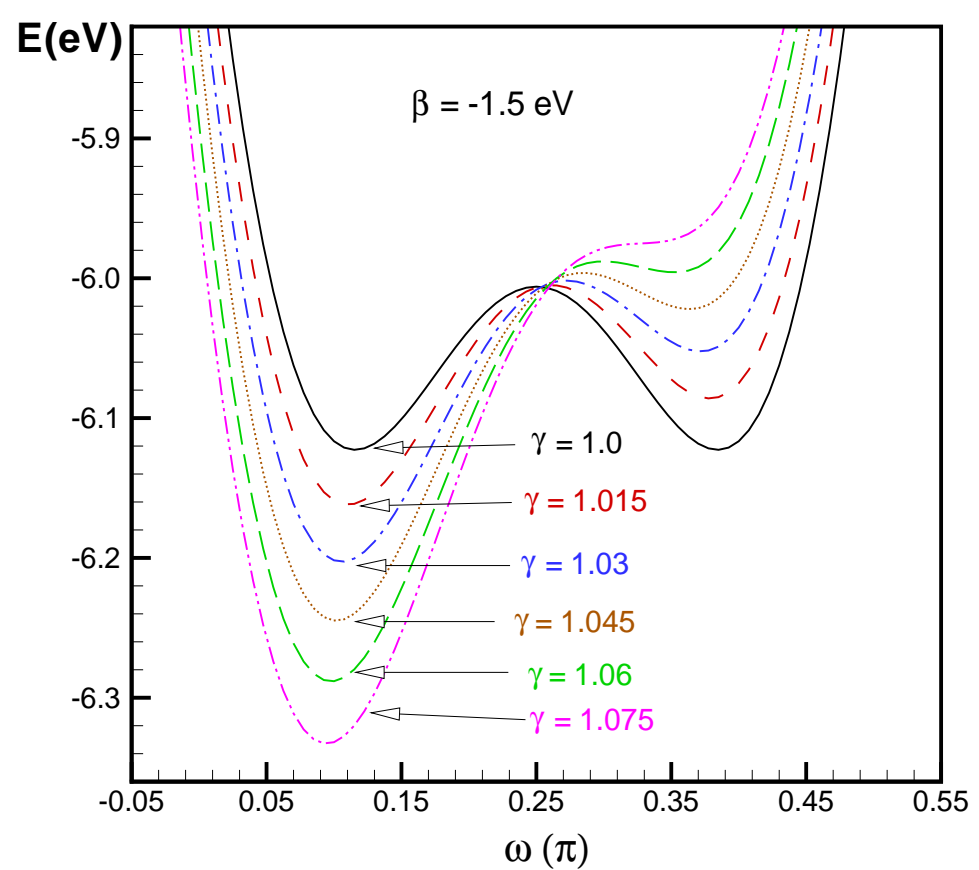

Figure 6: Mean energy value functional $E_{\text {tot }}(\omega)$, Eq. (41), dependence on the variational parameter $\omega$ for several values of the distortion parameter $\gamma$, Eq. (40), and $\beta=-1.5 \mathrm{eV}$. The plots are shifted to coincide at $\omega=\pi / 4$. Clearly, for $\gamma<1$ we obtain identical plots that are reflected about the $\omega=\pi / 4$ axis (cf. also Fig. 7 ).

derivative $E_{\text {tot }}^{\prime}(\omega)$ dependence by locating the sign changes in the appropriate list or plot. To get a precise value we can interpolate between a few points surrounding these roots. The second derivative $E_{\text {tot }}^{\prime \prime}\left(\omega_{\text {opt }}\right)$ then verifies the nature of the extremum, a positive value for a minimum and a negative one for a maximum. In the critical case for $\Delta=\Delta_{\text {crit }}$ it will identify the inflexion point. 


\section{Discussion and Conclusions}

Using a simple $\pi$-electron model of the allyl radical as described by the PPP Hamiltonian we were able to test the HF doublet-stability conditions by comparing the resulting critical value for the resonance integral $\beta, \beta_{\text {crit }}=-\left(\gamma_{0}-\gamma_{2}\right) /(2 \sqrt{2})=-2.48$ $\mathrm{eV}$, with the result obtained by direct analytical derivation of the explicit expressions for the mean energy value and its derivatives [cf. Eqs. (24) and (35)]. Moreover a similar explicit description was possible for HF solutions that are associated with distorted structures of our model. These solutions are of particular interest for the region of a coupling constant in which the symmetry adapted HF solution is doublet unstable implying the existence of lower-lying BS HF solutions of both the first and the second kind (the latter for correspondingly distorted structures).

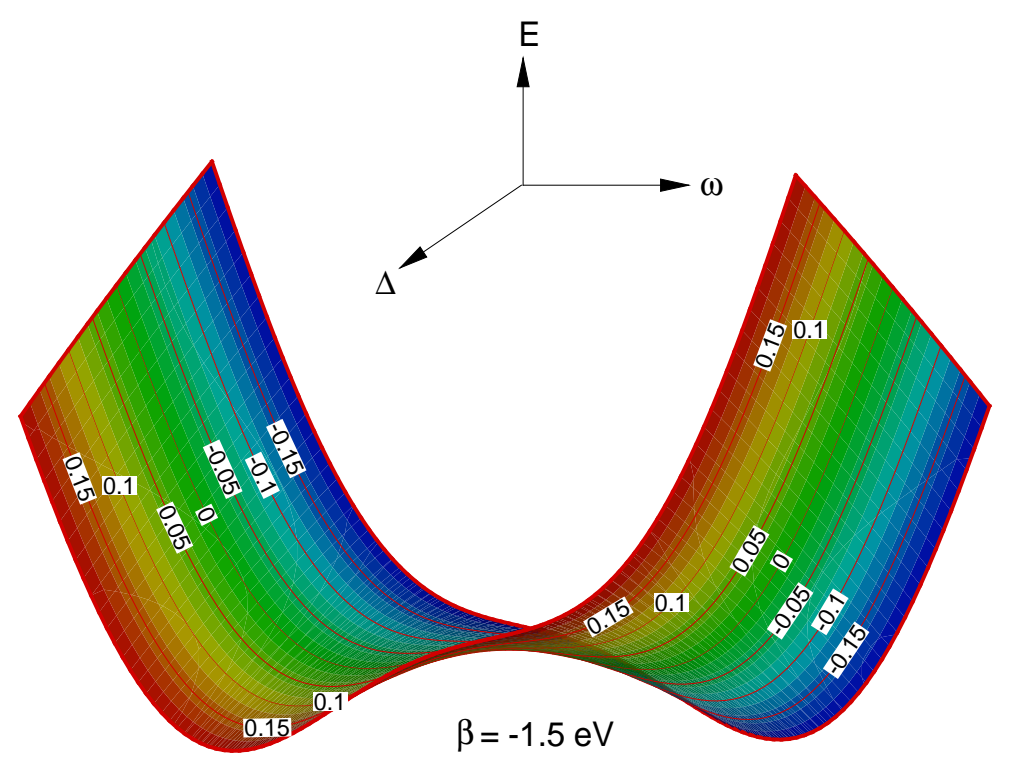

Figure 7: Variational surface $E_{\text {tot }}(\omega)$, Eq. (41), for distorted structures as a function of both the $\omega$ and $\Delta$ parameters for $\beta=-1.5 \mathrm{eV}$.

Using the explicit energy expressions for the total energy and its derivatives, Eqs. (41) to (43), we were thus able to find easily relevant HF solutions for any set of parameters defining our PPP Hamiltonian as is apparent from the plots of the mean energy values $E_{\text {tot }}(\omega)$ as a function of the variational parameter $\omega$ (cf. Fig. 6). The entire energy surface as a function of both the distortion parameter $\Delta$ and the variational parameter $\omega$ for a typical value of the resonance integral $\beta=-1.5 \mathrm{eV}$ is displayed in Fig. 7. The same surface for resonance integral values of -2 and $-1 \mathrm{eV}$ is also shown, respectively, in Figs. 8 and 9 as an iso-energy plot. Based on these results we can generate the required PES or its various cuts. 


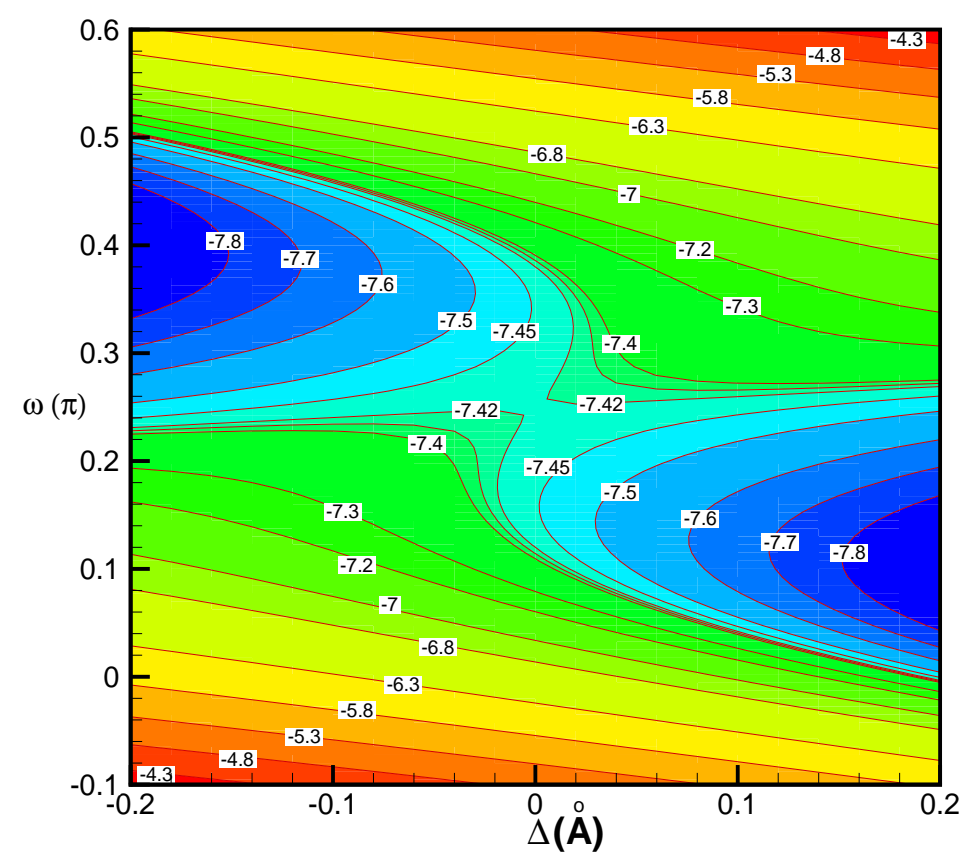

Figure 8: Energy levels representing the surface shown in Fig. 7 for $\beta=-2 \mathrm{eV}$.

A particularly revealing cut of the PES is along the line $d_{1}+d_{2}=2 d$ which is perpendicular to the "breezing mode" coordinate given by the line $d_{1}=d_{2}$ representing symmetric structures (cf. Fig. 3 of [5]). Several of such PES cuts for different values of the resonance integral are shown in Fig. 10 revealing a typical swallowtail shape characterizing the singular nature of this part of the PES. In this figure the dots indicate the energy of unstable symmetry-adapted ROHF solutions while the PECs intersect at the energy of the degenerate BS ROHF solutions for $\Delta=0$. The dotted lines indicate the energy of unstable ROHF solutions as given by the maxima on the mean energy value plots (cf. Fig. 6).

A 3D plot of the relevant part of the PES displaying its singular behavior in the vicinity of the critical $\beta$ value $(-3.5 \mathrm{eV} \leqslant \beta \leqslant-1.0 \mathrm{eV})$ is shown in Fig. 11 as a function of $\beta$ and $\Delta$. To achieve a more conspicuous representation we have shifted the energies relative to the energy of BS solutions at $\Delta=0$. The parts of the PES lying, respectively, below and above (the latter for $\beta>\beta_{\text {crit }}$ ) the $E(\Delta=0)$ energy (orange and violet online) are associated with stable ROHF solutions, while the part that is associated with unstable ROHF solutions is represented by the topmost, triangularshaped surface (green online) joining the stable solution surfaces situated above the $E(\Delta=0)$ energy reference. The figure also displays the cuts across the PES for the limiting values of $\beta$, namely $\beta=-1$ and $-3.5 \mathrm{eV}$, as well as for the critical $\beta$ value at $\beta=\beta_{\text {crit }} \approx-2.48 \mathrm{eV}$. The latter delineates the region of the existence of unstable $\mathrm{HF}$ solutions. Note that the cut at $\beta=-1 \mathrm{eV}$ is identical with that displayed in 


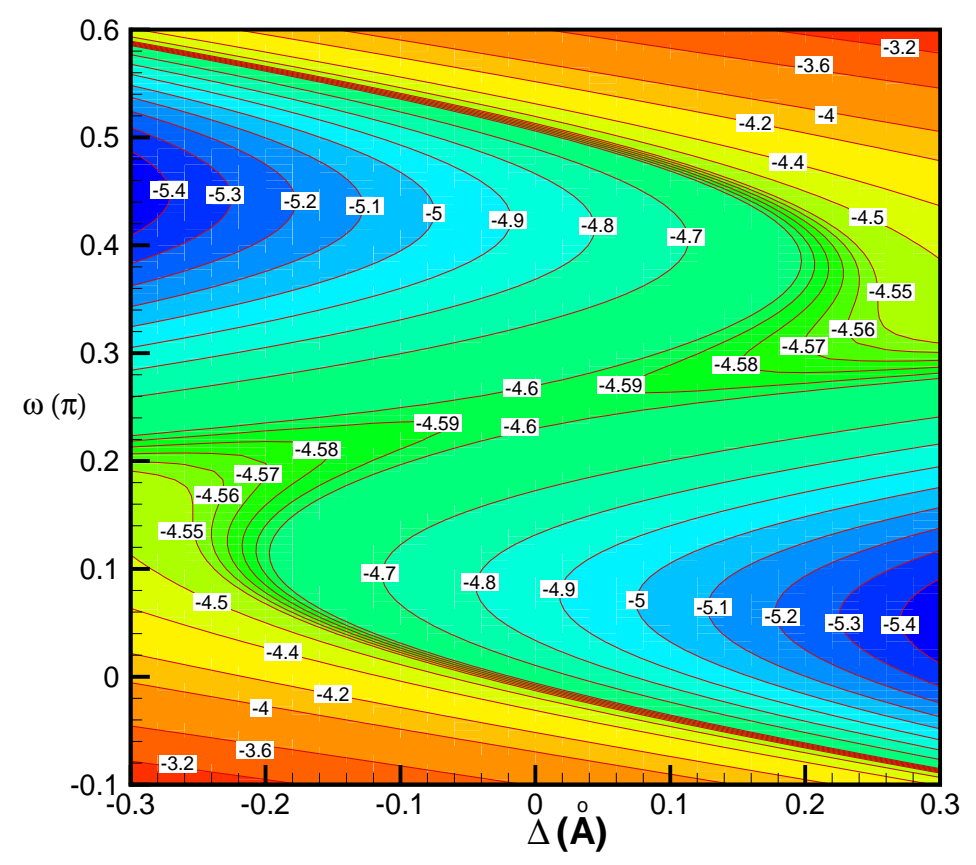

Figure 9: Energy levels representing the surface shown in Fig. 7 for $\beta=-1 \mathrm{eV}$.

Fig. 10 for the same $\beta$ value. It should be noted that in the case of our simple model we can just as easily determine the energies of unstable HF solutions for distorted structures as for the stable ones. These unstable solutions exist within the interval of distortion parameters $\Delta \in\left[-\Delta_{\text {crit }}, \Delta_{\text {crit }}\right]$, with $\Delta_{\text {crit }}$ defined by the distortion for which the maximum and one of the minima on the $E_{\text {tot }}(\omega)$ plot merge into an inflexion point (cf. Fig. 6).

Note also that PESs in Fig. 11 that are associated with stable ROHF solutions intersect in a line corresponding to degenerate BS solutions at $\Delta=0$ or $\gamma=1$ and their extensions beyond this point eventually disappear at $\Delta= \pm \Delta_{\text {crit }}$ where they join with those associated with unstable solutions, thus yielding a complete representation of the relevant swallow-tail singularity. In other more complex situations it is extremely difficult, if not impossible, to generate that part of the PES that is associated with unstable solutions. Even a very careful "analytic continuation" starting at the doubly degenerate BS solution soon breaks down.

We can conclude that the studied simple model of the allyl radical demonstrates both the appropriateness of the doublet-stability conditions for ROHF solutions and enables a full description of the singular nature of the resulting ROHF PESs. 


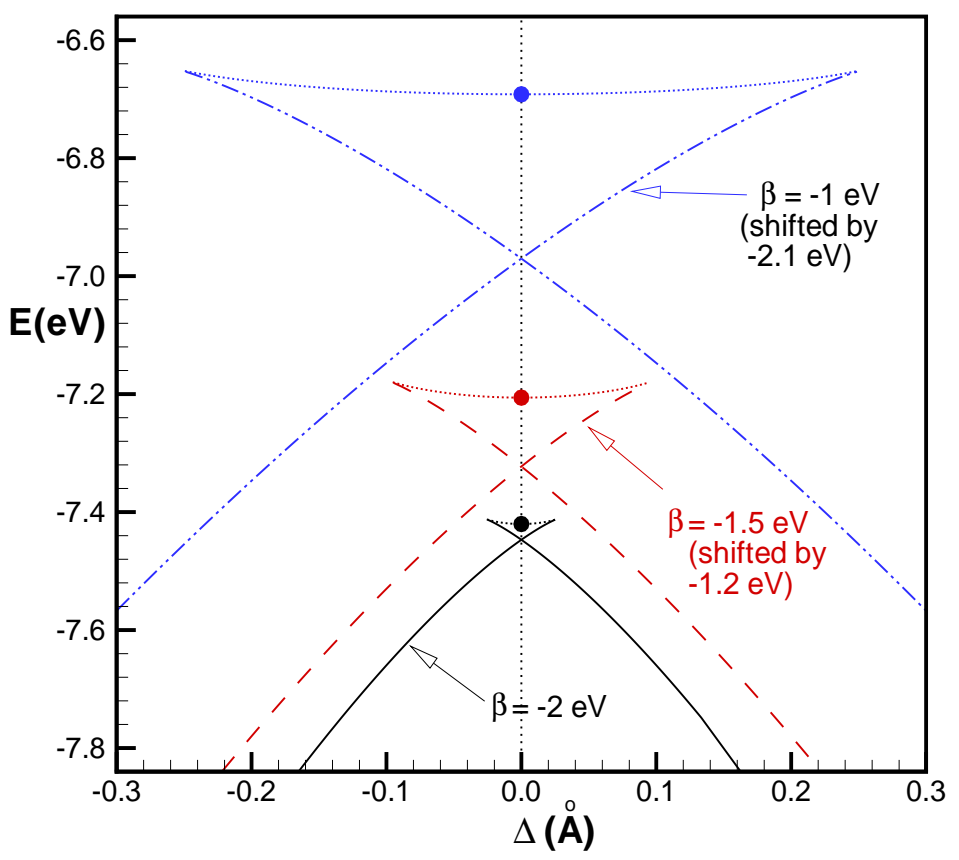

Figure 10: Cuts of the PES along the line $d_{1}+d_{2}=2 d$ as a function of the distortion parameter $\Delta=d_{1}-d_{2}$ for several values of the resonance integral $\beta$ showing a typical swallowtail singularity.

Acknowledgments Two of the authors (J.P. and T.S.) are greatly indebted to the Alexander von Humboldt Foundation for its kind support that enabled their stay at the Max-Planck Institute for Astrophysics in Garching bei München in Germany and they thank the latter Institute for its hospitality during their stay. Their heartfelt thanks are also due to their host, Prof. Dr. Geerd H. F. Diercksen, for his kind advice and collaborations and for making their stay as pleasant and productive as possible. 


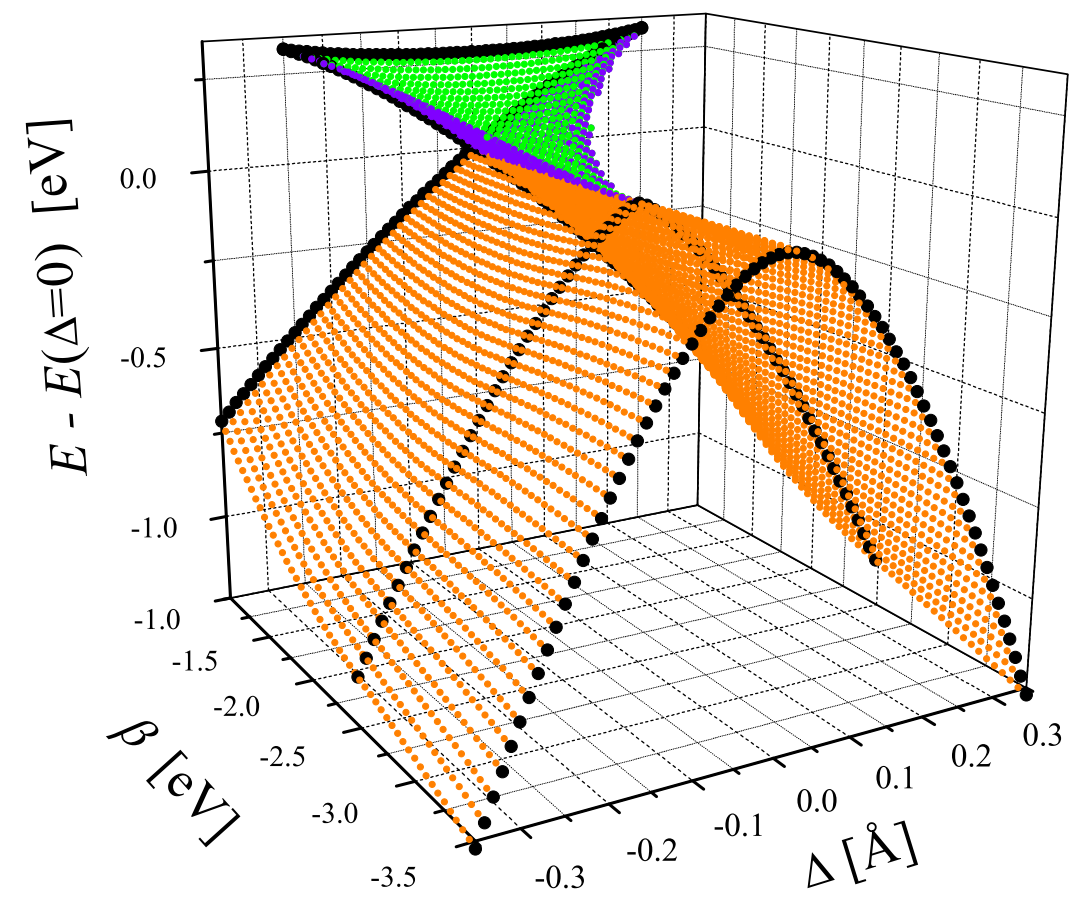

Figure 11: A 3D plot of the relevant part of the potential energy surface (PES) as a function of both $\beta$ and $\gamma$ parameters in the vicinity of the critical $\beta$ value $(-3.5 \mathrm{eV}$ $\leqslant \beta \leqslant-1.0 \mathrm{eV}$ ) displaying the character of the "swallow-tail" singularities arising for $|\beta|<\mid \beta_{\text {crit }}$.

\section{Appendix: One- and two-electron integrals}

We present below the expressions for the required one- and two-electron integrals over the MOs (25) for general distorted structures. The corresponding expressions for the symmetric structures are obtained by identifying primed and unprimed parameters, i.e., by setting $\beta=\beta^{\prime}$ and $\gamma_{1}=\gamma_{1}^{\prime}$.

$$
\begin{aligned}
\langle 1|z| 1\rangle & =\beta s+\beta^{\prime} c-\frac{1}{2} \gamma_{1}\left(1+s^{2}\right)-\frac{1}{2} \gamma_{1}^{\prime}\left(1+c^{2}\right)-\frac{1}{2} \gamma_{2} \\
\langle 0|z| 0\rangle & =-\gamma_{1} c^{2}-\gamma_{1}^{\prime} s^{2}-\gamma_{2} \\
\langle 11|v| 11\rangle & =\frac{1}{4} \gamma_{0}\left(1+s^{4}+c^{4}\right)+\frac{1}{2}\left(\gamma_{1} s^{2}+\gamma_{1}^{\prime} c^{2}+\gamma_{2} s^{2} c^{2}\right) \\
\langle 10|v| 10\rangle & =\gamma_{0} s^{2} c^{2}+\frac{1}{2}\left[\gamma_{1} c^{2}+\gamma_{1}^{\prime} s^{2}+\gamma_{2}\left(s^{4}+c^{4}\right)\right] \\
\langle 10|v| 01\rangle & =\left(\gamma_{0}-\gamma_{2}\right) s^{2} c^{2}
\end{aligned}
$$




\section{References}

[1] J. Paldus, A. Veillard, Chem. Phys. Lett. 50, 6 (1977)

[2] J. Paldus, A. Veillard, Mol. Phys. 35, 445 (1978)

[3] M. Bénard, J. Chem. Phys. 71, 2546 (1979)

[4] M. Bénard, J. Paldus, J. Chem. Phys. 72, 6546 (1980)

[5] J. Paldus, E. Chin, Int. J. Quantum. Chem.24, 373 (1983)

[6] J. Paldus, J. Č́ížek, Can. J. Chem. 63, 1803 (1985)

[7] M. Bénard, W. G. Laidlaw, J. Paldus, Can. J. Chem. 63, 1797 (1985)

[8] M. Bénard, W. G. Laidlaw, J. Paldus, Chem. Phys. 103, 43 (1986)

[9] X. Li, J. Paldus, J. Chem. Phys. 126, 224304 (2007)

[10] F. Holka, P. Neogrády, M. Urban, J. Paldus, Collect. Czech. Chem. Commun. 72, 197 (2007)

[11] X. Li, J. Paldus, Int. J. Quantum. Chem. 108, 2117 (2008)

[12] J. Paldus, G. Thiamová, J. Math. Chem. 44, 88 (2008)

[13] G. Thiamová, J. Paldus, Eur. Phys. J. D 46, 453 (2008)

[14] X. Li, J. Paldus, Int. J. Quantum. Chem. 109, 1756 (2009)

[15] X. Li, J. Paldus, J. Chem. Phys. 130, 084110 (2009)

[16] X. Li, J. Paldus, Phys. Chem. Chem. Phys. 11, 5281 (2009)

[17] X. Li, J. Paldus, J. Chem. Phys. 130, 164116 (2009)

[18] H. Fukutome, Int. J. Quantum. Chem. 20, 955 (1981)

[19] J. Paldus, in Self-Consistent Field: Theory and Applications, ed. by R. Carbó, M. Klobukowski (Elsevier, Amsterdam, 1990) pp. 1-45

[20] M. M. Mestechkin, in Self-Consistent Field: Theory and Applications, ed. by R. Carbó, M. Klobukowski, (Elsevier, Amsterdam, 1990) pp. 312-385

[21] J.L. Stuber, J. Paldus, in Fundamental World of Quantum Chemistry, A Tribute Volume to the Memory of Per-Olov Löwdin, ed. by R. J. Brändas, E. S. Kryachko, vol. I (Kluwer, Dordrecht, 2003) pp. 67-139

[22] D. J. Thouless, Nucl. Phys. 21, 225 (1960)

[23] J. Č́žzek, J. Paldus, J. Chem. Phys. 47, 3976 (1967) 
[24] J. Paldus, J. Čížek, Progr. Theor. Phys. (Kyoto) 42, 769 (1969)

[25] J. Paldus, J. Čížek, Phys. Rev. A 2, 2268 (1970)

[26] J. Č́žžek, J. Paldus, Phys. Rev. A 3, 525 (1971)

[27] J. Paldus, J. Čížek, Chem. Phys. Lett. 3, 1 (1969)

[28] J. Paldus, J. Čížek, J. Chem. Phys. 52, 2919 (1970)

[29] J. Č́ížek, J. Paldus, J. Chem. Phys. 53, 821 (1970)

[30] J. Paldus, J. Čížek, J. Chem. Phys. 54, 2293 (1971)

[31] H. Fukutome, Progr. Theoret. Phys. (Kyoto) 45, 1382 (1971)

[32] H. Fukutome, Progr. Theoret. Phys. (Kyoto) 52, 115 (1974)

[33] H. Fukutome, Progr. Theoret. Phys. (Kyoto) 52, 1766 (1974)

[34] H. Fukutome, Progr. Theoret. Phys. (Kyoto) 53, 1320 (1975)

[35] M. Ozaki, Progr. Theoret. Phys. (Kyoto) 67, 415 (1982) and references therein

[36] P.-O. Löwdin, Rev. Mod. Phys. 35, 496 (1963)

[37] J. Paldus, J. Čížek, B. A. Keating, Phys. Rev. A 8, 640 (1973)

[38] X. Li, J. Paldus, J. Chem. Phys. bf 134, 074301 (2011)

[39] J. Paldus, E. Chin, M. G. Grey, Int. J. Quantum. Chem. 24, 395 (1983)

[40] M. Takahashi, J. Paldus, Int. J. Quantum. Chem. 26, 349 (1984)

[41] M. Takahashi, J. Paldus, Can. J. Phys. 62, 1226 (1984)

[42] J. Paldus, M. Takahashi, R. W. H. Cho, Phys. Rev. B 30, 4267 (1984)

[43] M. Takahashi, J. Paldus, Phys. Rev. B 31, 5121 (1985)

[44] C. Yannouleas, U. Landman, Rep. Progr. Phys. 70, 2067 (2007)

[45] T. Ihn, C. Ellenberger, K. Ensslin, C. Yannouleas, U. Landman, D. C. Driscoll, A. C. Gossard, Int. J. Modern Phys. B 21, 1316 (2007)

[46] K. Kowalski, K. Jankowski, Phys. Rev. Lett. 81, 1195 (1998)

[47] J. Paldus, in Theoretical Chemiatry: Advances and Perspectives, ed. by $\mathrm{H}$. Eyring, D. J. Henderson, vol. 2 (Academic, New York, 1976) pp. 131-290

[48] M. Goeppert-Mayer and A. L. Sklar, J. Chem. Phys. 6, 635 (1938)

[49] R. G. Parr, The Quantum Theory of Molecular Electronic Structure (Benjamin, New York, 1963) 
[50] N. Mataga, K. Nishimoto, Z. Phys. Chem. (Frankfurt) 13, 140 (1957)

[51] J. Koutecký, J. Paldus, R. Zahradník, J. Chem. Phys. 36, 3129 (1962)

[52] A. Laforgue, J. Č́žek, J. Paldus, J. Chem. Phys.59, 2560 (1973).

[53] J. Koutecký, J. Paldus, J. Č́žžek, J. Chem. Phys. 83, 1722 (1985) and references therein. 